Clinical Study

\title{
Baseline Obesity Status Modifies Effectiveness of Adapted Diabetes Prevention Program Lifestyle Interventions for Weight Management in Primary Care
}

\author{
Kristen M. J. Azar, ${ }^{1}$ Lan Xiao, ${ }^{1}$ and Jun $\mathrm{Ma}^{1,2}$ \\ ${ }^{1}$ Palo Alto Medical Foundation Research Institute, 795 El Camino Real, Ames Building, Palo Alto, CA 94301, USA \\ ${ }^{2}$ Stanford Prevention Research Center, Department of Medicine, Stanford University School of Medicine, Stanford, CA, USA \\ Correspondence should be addressed to Kristen M. J. Azar; azark@pamfri.org
}

Received 30 September 2013; Accepted 13 November 2013

Academic Editor: Pierpaolo De Feo

Copyright ( 2013 Kristen M. J. Azar et al. This is an open access article distributed under the Creative Commons Attribution License, which permits unrestricted use, distribution, and reproduction in any medium, provided the original work is properly cited.

\begin{abstract}
Objective. To examine whether baseline obesity severity modifies the effects of two different, primary care-based, technologyenhanced lifestyle interventions among overweight or obese adults with prediabetes and/or metabolic syndrome. Patients and Methods. We compared mean differences in changes from baseline to 15 months in clinical measures of general and central obesity among participants randomized to usual care alone $(n=81)$ or usual care plus a coach-led group $(n=79)$ or self-directed individual ( $n=81$ ) intervention, stratified by baseline body mass index (BMI) category. Results. Participants with baseline BMI 35+ had greater reductions in mean BMI, body weight (as percentage change), and waist circumference in the coach-led group intervention, compared to usual care and the self-directed individual intervention $(P<0.05$ for all). In contrast, the self-directed intervention was more effective than usual care only among participants with baseline BMIs between $25 \leq 35$. Mean weight loss exceeded $5 \%$ in the coach-led intervention regardless of baseline BMI category, but this was achieved only among self-directed intervention participants with baseline BMIs $<35$. Conclusions. Baseline BMI may influence behavioral weight-loss treatment effectiveness. Researchers and clinicians should take an individual's baseline BMI into account when developing or recommending lifestyle focused treatment strategy. This trial is registered with ClinicalTrials.gov (NCT00842426).
\end{abstract}

\section{Introduction}

Obesity remains a pressing public health problem with adverse medical, psychological, social, and economical consequences. Nearly $70 \%$ of US adults are overweight (body mass index $[\mathrm{BMI}]$ in $\left.\mathrm{kg} / \mathrm{m}^{2} 25 \leq 30\right)$ or obese $(\mathrm{BMI} \geq 30)$, with $36 \%$ obese [1]. More alarming still, the $6.3 \%$ prevalence of severely obesity (BMI $\geq 40$ ) [1] is projected to increase by $130 \%$ over the next 2 decades [2]. Although bariatric surgery is the recommended treatment for severely obese individuals and/or moderately obese individuals (BMI $35 \leq$ 40) with comorbidities [3], its implementation is limited by access, cost, recidivism, and complications $[4,5]$. Only $1 \%$ $2 \%$ of obese people eligible for insurance coverage of surgical treatment receive it, compelling an urgent need of alternative treatment strategies for this subpopulation [6]. Weight loss medications have had limited effectiveness, some serious adverse effects, and limited uptake [7].

Emerging data find intensive lifestyle interventionsfocusing on calorie-reduced, healthful eating, increased physical activity, and self-management skills training-can lead to clinically significant weight loss in the short [8-10] and long term $[10,11]$ among individuals with a $\mathrm{BMI} \geq 35$, who also achieve improvements in cardiovascular disease (CVD) risk factors even despite persistent, albeit reduced, obesity after intervention. Despite a recent and renewed interest in examining the efficacy of intensive behavior therapy for obesity within higher BMI subcategories [12], very few studies have evaluated the comparative effectiveness of evidence-based, empirical lifestyle interventions in realworld settings by baseline obesity status. Implementation of efficacious but resource-intensive, research-based lifestyle 
TABLE 1: E-LITE ${ }^{\mathrm{a}}$ intervention: key components and features.

\begin{tabular}{|c|c|c|}
\hline & Coach-led intervention & Self-directed intervention \\
\hline (1) 12-week core curriculum sessions ${ }^{b}$ & Clinic-based, small groups & Home-based DVD \\
\hline $\begin{array}{l}\text { (2) Online self-monitoring of weight and } \\
\text { physical activity }^{c}\end{array}$ & $\begin{array}{l}\text { Preferably daily but at least twice weekly; } \\
\text { coach routinely reviewed records }\end{array}$ & $\begin{array}{l}\text { Preferably daily but at least twice weekly; } \\
\text { coach did not routinely review records }\end{array}$ \\
\hline (3) Personalized lifestyle coaching ${ }^{\mathrm{d}}$ & Proactive, coach-initiated & As needed, patient-initiated \\
\hline
\end{tabular}

${ }^{a}$ E-LITE: Evaluation of lifestyle interventions to treat elevated cardiometabolic risk in primary care.

${ }^{b}$ Diabetes Prevention Program (DPP) investigators at the University of Pittsburgh developed the Group Lifestyle Balance (GLB) program following the DPP trial [13]. Its curriculum is publicly available online [14].

${ }^{c}$ Via the American Heart Association's free, secure Heart360 Web portal (http://www.heart360.org/).

${ }^{\mathrm{d}}$ Via secure provider-patient online messaging embedded in a fully functional electronic health record system. Coaches could view Heart360 patient selfmonitoring records, which they reviewed regularly and used to tailor their ongoing progress feedback via secure messaging for participants in the coach-led intervention.

interventions into real-world settings remains a challenge. Efforts have been made to facilitate this process while retaining essential components of efficacious interventions [13]. However, these same efforts have resulted in wide variation in intervention setting, structure, intensity and form of contact, and resources required-and (unsurprisingly) they have produced mixed results regarding clinical effectiveness. Improved ability to implement targeted interventions for readily defined subgroups of the intended population may result in more efficient and effective use of resources.

The "Evaluation of Lifestyle Interventions to Treat Elevated Cardiometabolic Risk in Primary Care" (E-LITE) study was one of few pragmatic randomized controlled trials that successfully translated the Diabetes Prevention Program (DPP) lifestyle intervention into a primary care setting in the US. Published E-LITE data have demonstrated that two adapted, technology-enhanced DPP interventions (further described in Section 2)-one using a self-directed approach and the other a coach-led approach-were both superior to usual care, whereas the coach-led intervention was superior to the self-directed one, in promoting weight loss among overweight or obese adults with prediabetes and/or metabolic syndrome [15]. The primary aim of the current study was to examine whether changes in clinical measures of general and abdominal obesity differed by baseline BMI category when comparing the two interventions to usual care and to each other. We hypothesized that baseline BMI modified participant response to treatment such that participants with baseline BMI 35+ would benefit from the more structured, coach-led intervention, whereas those with lower starting BMI would respond to either coach-led or self-directed intervention.

\section{Materials and Methods}

Complete E-LITE trial protocol and methods were published previously [16]. Data collection occurred in 2009-2011. Below we describe methodological details relevant to this study.

2.1. Study Population and Measures. Participants were recruited (July 2009-June 2010) from a single primary care clinic that is part of a large multispecialty group practice in the San Francisco Bay Area. All data collection and intervention visits occurred at the clinic. Inclusion criteria included an age of at least 18 years, a BMI of at least 25, and the presence of prediabetes (defined by impaired fasting plasma glucose level of 100 to $125 \mathrm{mg} / \mathrm{dL}$ ) or metabolic syndrome. Major exclusion criteria included serious medical or psychiatric conditions (e.g., stroke, psychotic disorder) or special life circumstances (e.g., pregnancy). Eligible and consenting overweight or obese adults with prediabetes and/or metabolic syndrome seen in primary care were randomized to receive usual care alone $(n=81)$ or usual care plus a coach-led $(n=79)$ or self-directed $(n=81)$ behavioral weight-loss intervention. Height was measured at baseline only, and weight and waist circumference were measured at baseline and at 3, 6, and 15 months. Measurements were taken in duplicate per standardized protocols $[17,18]$. Body mass index was calculated. Change in BMI from baseline to 15 months was the trial primary outcome [15].

2.2. Intervention. The E-LITE study innovatively integrated the DPP-based Group Lifestyle Balance (GLB) core curriculum [13], which has been recognized by the Centers for Disease Prevention and Control's National Diabetes Prevention Program, with lifestyle coaching and self-management support via high-reach, affordable technologies. Both E-LITE interventions adopted the DPP's weight loss and physical activity goals [19] and delivered the GLB core curriculum for 12 weeks during the intensive treatment phase either through a self-directed, take-home DVD or coach-led, inclinic small groups. The interventions also provided electronically mediated lifestyle coach contact and online selfmonitoring of weight and physical activity goal attainment during a 12-month maintenance phase. Table 1 summarizes the key components and features of the E-LITE self-directed and coach-led interventions.

2.3. Statistical Analyses. Baseline characteristics of each study group by baseline BMI category $(25 \leq 30,30 \leq 35$, or $35+$ ) were examined using analysis of variance (ANOVA) for continuous variables and Chi-square test for categorical variables. Between-group differences by baseline BMI category at 15 months were evaluated by intention-to-treat using all available data and tests of group by baseline BMI category interactions in repeated-measures mixed models. A 


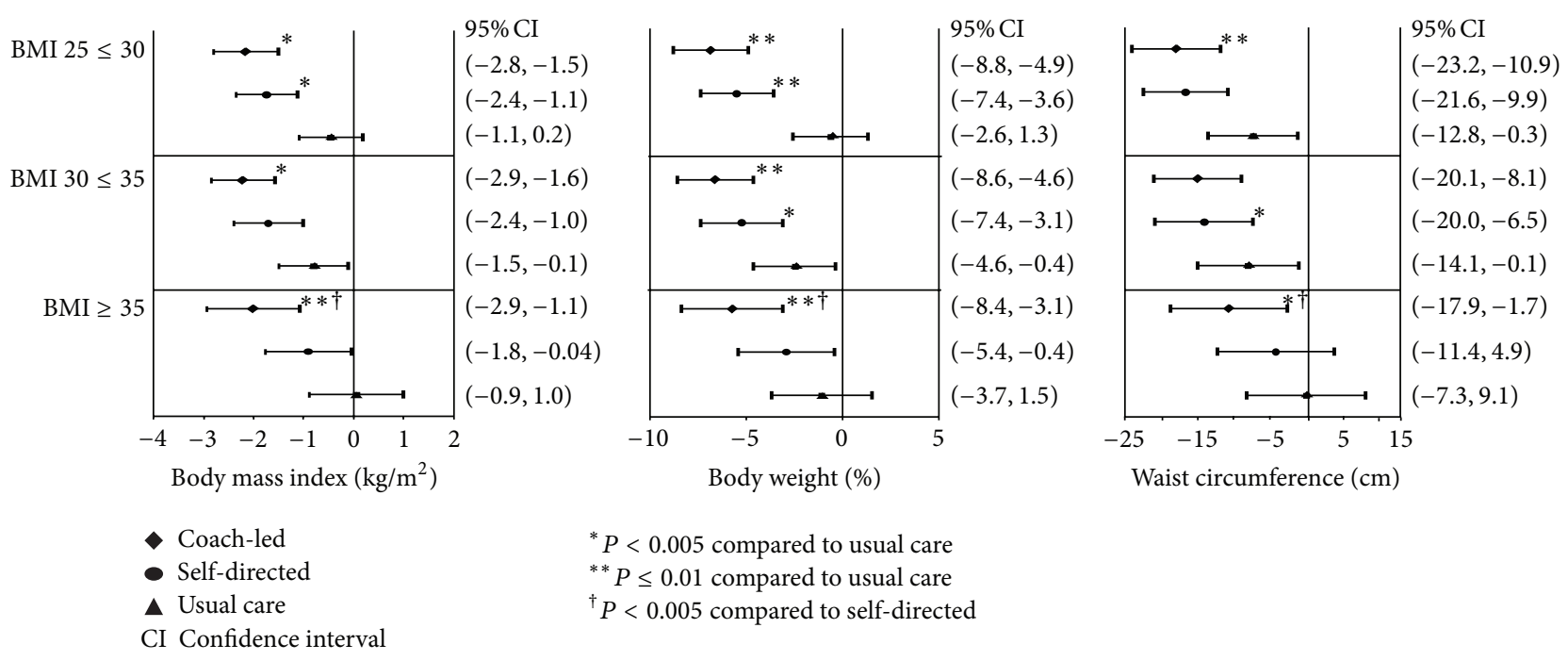

FIgURE 1: Outcomes by baseline body mass index category and intervention type.

separate model examined change in each of the 3 obesity outcome variables: BMI and percent body weight change for general obesity and waist circumference for abdominal obesity. The group by baseline BMI category interaction terms were significant for all three outcomes $(P<0.001)$. As in the main study [15], these models were adjusted for age, sex, race, and ethnicity, and missing data were handled directly through maximum likelihood estimation via mixed modeling. Model-based least-square mean changes and 95\% confidence intervals (CIs) were obtained. All analyses were conducted using SAS, version 9.2 (SAS Institute Inc., Cary, North Carolina).

\section{Results and Discussion}

3.1. Results. Table 2 shows baseline sample characteristics, stratified by baseline BMI. No significant differences in baseline characteristics were detected between participants in each treatment group. The coach-led intervention resulted in significantly greater mean reductions from baseline to 15 months in BMI (ranging from $-2.0 \mathrm{~kg} / \mathrm{m}^{2}, 95 \%$ CI $\left[-2.9 \mathrm{~kg} / \mathrm{m}^{2},-1.1 \mathrm{~kg} / \mathrm{m}^{2}\right]$ in the BMI $35+$ category to $-2.2 \mathrm{~kg} / \mathrm{m}^{2}, 95 \% \mathrm{CI}\left[-2.9 \mathrm{~kg} / \mathrm{m}^{2},-1.6 \mathrm{~kg} / \mathrm{m}^{2}\right]$ in the BMI $30 \leq$ 35 category) and percent body weight change (ranging from $-6.8 \%, 95 \%$ CI $[-8.8 \%,-4.9 \%]$ in the BMI $25 \leq 30$ category to $-5.7 \%, 95 \% \mathrm{CI}[-8.4 \%,-3.1 \%]$ in the BMI $35+$ category) for all three baseline BMI categories, and in waist circumference for the BMI $25 \leq 30(-17.1 \mathrm{~cm}, 95 \%$ CI $[-23.2 \mathrm{~cm},-10.95 \mathrm{~cm}])$ and $35+(-9.78 \mathrm{~cm}, 95 \% \mathrm{CI}[-17.9 \mathrm{~cm},-1.7 \mathrm{~cm}])$ categories $(P<0.05$ versus usual care for all; Figure 1). The coachled group achieved a mean percentage weight loss exceeding $5 \%$, a commonly accepted threshold of clinically significant weight loss, and the upper $95 \%$ confidence limit was at least $3 \%$ weight loss, across the baseline BMI categories.

The self-directed intervention led to greater improvements in BMI ( $P=0.03$ versus usual care $)$ only for the BMI $25 \leq 30$ category $\left(-1.7 \mathrm{~kg} / \mathrm{m}^{2}, 95 \%\right.$ CI $\left[-2.4 \mathrm{~kg} / \mathrm{m}^{2}\right.$, $\left.-1.1 \mathrm{~kg} / \mathrm{m}^{2}\right]$ ), in percentage weight loss for the BMI $25 \leq 30$ $(-5.5 \%, 95 \%$ CI $[-7.4 \%,-3.6 \%] ; P<0.0001$ versus usual care) and $30 \leq 35(-5.2 \%, 95 \%$ CI $[-7.4 \%,-3.1 \%] ; P=0.02$ versus usual care) categories, and in waist circumference for the BMI $30 \leq 35$ category $(-13.2 \mathrm{~cm}, 95 \%$ CI $[-20.0 \mathrm{~cm}$, $-8.1 \mathrm{~cm}] ; P=0.03)$. In the self-directed group mean weight loss reached 5\% only among those with a baseline BMI of $<35$. Moreover, reductions in BMI $(P=0.01)$, weight as percentage change $(P=0.04)$, and waist circumference $(P=$ 0.04 ) were significantly greater in the coach-led versus selfdirected intervention within the BMI 35+ category, whereas the two interventions did not differ significantly for any of the three obesity measures in the two lower BMI categories.

3.2. Discussion. Efficacy research has unequivocally shown that intensive, highly structured, individual lifestyle intervention lowers cardiometabolic risk [20]. The unabated obesity epidemic and its associated health problems and rising societal and economical burdens compel the urgency of adapting proven, albeit expensive, interventions into increasingly resource-limited real-world settings while striving to retain the effectiveness of the original treatment. The current findings show that the effects of the successful E-LITE coachled and self-directed interventions in primary care differed by starting obesity status, suggesting that one size may not fit all when it comes to lifestyle interventions.

Notably participants with moderate or severe obesity (baseline BMI 35+) had greater reductions in all three obesity measures (BMI, percentage weight loss, and waist circumference) in the coach-led intervention, but not in the self-directed intervention, compared with usual care. They also responded more favorably to the coach-led intervention compared to the self-directed intervention. In contrast, overweight participants (baseline BMI $25 \leq 30$ ) had similar mean BMI and percent body weight reductions in the two active interventions, both of which were more effective than usual care. Similarly, the coach-led intervention had no apparent 
TABLE 2: Baseline characteristics of the study participants ${ }^{\mathrm{a}}$.

\begin{tabular}{|c|c|c|c|c|c|c|}
\hline Characteristic & All & Usual care & Coach-led & Self-directed & $F / \chi^{2}$ (degree of freedom) & $P$ value \\
\hline \multicolumn{7}{|l|}{ Body mass index $25 \leq 30$} \\
\hline Age, year & $53.8 \pm 10.5$ & $53.7 \pm 10.3$ & $54.7 \pm 10.9$ & $53.1 \pm 10.6$ & $0.22(2,108)$ & 0.80 \\
\hline Female, $\%$ & 32.4 & 32.4 & 33.3 & 31.6 & $0.03(2)$ & 0.99 \\
\hline Race/ethnicity, \% & & & & & $3.29(6)$ & 0.77 \\
\hline Non-Hispanic white & 72.1 & 75.7 & 66.7 & 73.7 & & \\
\hline Asian/Pacific Islander & 22.5 & 18.9 & 27.8 & 21.1 & & \\
\hline Latino/Hispanic & 4.5 & 5.4 & 5.6 & 2.6 & & \\
\hline College level or above, $\%$ & 97.2 & 97.2 & 94.3 & 100.0 & $2.22(2)$ & 0.33 \\
\hline Income, $\%$ & & & & & $6.62(6)$ & 0.36 \\
\hline$<\$ 75,000$ & 10.3 & 13.9 & 8.8 & 8.1 & & \\
\hline$\$ 75,000-\$ 124,999$ & 30.8 & 27.8 & 41.2 & 24.3 & & \\
\hline$\$ 125,000-\$ 149,999$ & 15.9 & 8.3 & 20.6 & 18.9 & & \\
\hline$\$ 150,000+$ & 43.0 & 50.0 & 29.4 & 48.6 & & \\
\hline Weight, kg & $83.8 \pm 9.9$ & $85.4 \pm 9.1$ & $82.6 \pm 10.2$ & $83.5 \pm 10.6$ & $0.77(2,108)$ & 0.46 \\
\hline Waist, cm & $98.8 \pm 6.4$ & $98.3 \pm 6.4$ & $98.0 \pm 6.4$ & $100.1 \pm 6.4$ & $1.14(2,108)$ & 0.32 \\
\hline Fasting plasma glucose, $\mathrm{mg} / \mathrm{dL}$ & $100.3 \pm 9.3$ & $99.9 \pm 9.5$ & $101.4 \pm 9.3$ & $99.6 \pm 9.2$ & $0.39(2,108)$ & 0.68 \\
\hline Prediabetes, $\%$ & 56.8 & 62.2 & 61.1 & 47.4 & $2.08(2)$ & 0.35 \\
\hline Metabolic syndrome, $\%$ & 80.2 & 67.6 & 88.9 & 84.2 & $5.81(2)$ & 0.06 \\
\hline Prediabetes and metabolic syndrome, \% & 36.9 & 29.7 & 50.0 & 31.6 & $3.93(2)$ & 0.14 \\
\hline \multicolumn{7}{|l|}{ Body mass index $30 \leq 35$} \\
\hline Age, year & $54.0 \pm 10.8$ & $54.5 \pm 11.1$ & $55.3 \pm 12.9$ & $52.3 \pm 7.9$ & $0.51(2,72)$ & 0.60 \\
\hline Female, $\%$ & 50.7 & 44.0 & 50.0 & 58.3 & $1.01(2)$ & 0.60 \\
\hline Race/ethnicity, \% & & & & & $5.25(6)$ & 0.51 \\
\hline Non-Hispanic white & 80.0 & 80.0 & 80.8 & 79.2 & & \\
\hline Asian/Pacific Islander & 14.7 & 16.0 & 7.7 & 20.8 & & \\
\hline Latino/Hispanic & 4.0 & 4.0 & 7.7 & 0.0 & & \\
\hline College level or above, $\%$ & 97.3 & 96.0 & 96.2 & 100.0 & $0.97(2)$ & 0.62 \\
\hline Income, $\%$ & & & & & $5.16(6)$ & 0.52 \\
\hline$<\$ 75,000$ & 12.5 & 8.7 & 15.4 & 13.0 & & \\
\hline$\$ 75,000-\$ 124,999$ & 23.6 & 30.4 & 23.1 & 17.4 & & \\
\hline$\$ 125,000-\$ 149,999$ & 9.7 & 0.0 & 15.4 & 13.0 & & \\
\hline$\$ 150,000+$ & 54.2 & 60.9 & 46.2 & 56.5 & & \\
\hline Weight, kg & $94.2 \pm 13.8$ & $94.2 \pm 12.9$ & $97.2 \pm 14.5$ & $91.0 \pm 13.8$ & $1.26(2,72)$ & 0.29 \\
\hline Waist, $\mathrm{cm}$ & $106.7 \pm 8.1$ & $106.2 \pm 8.9$ & $109.2 \pm 7.1$ & $104.4 \pm 7.9$ & $2.35(2,72)$ & 0.10 \\
\hline Fasting plasma glucose, $\mathrm{mg} / \mathrm{dL}$ & $100.3 \pm 10.4$ & $98.7 \pm 8.6$ & $100.4 \pm 10.9$ & $101.8 \pm 11.6$ & $0.52(2,72)$ & 0.60 \\
\hline Prediabetes, \% & 50.7 & 44.0 & 50.0 & 58.3 & $1.01(2)$ & 0.60 \\
\hline Metabolic syndrome, $\%$ & 88.0 & 92.0 & 80.8 & 91.7 & $1.97(2)$ & 0.37 \\
\hline Prediabetes and metabolic syndrome, \% & 38.7 & 36.0 & 30.8 & 50.0 & $2.06(2)$ & 0.36 \\
\hline \multicolumn{7}{|l|}{ Body mass index $35+$} \\
\hline Age, year & $49.7 \pm 10.1$ & $47.4 \pm 10.6$ & $53.4 \pm 8.5$ & $48.6 \pm 10.4$ & $1.79(2,52)$ & 0.18 \\
\hline Female, $\%$ & 69.1 & 73.7 & 76.5 & 57.9 & $1.74(2)$ & 0.42 \\
\hline Race/ethnicity, \% & & & & & $2.58(4)$ & 0.63 \\
\hline Non-Hispanic white & 87.3 & 78.9 & 94.1 & 89.5 & & \\
\hline Asian/Pacific Islander & 9.1 & 15.8 & 5.9 & 5.3 & & \\
\hline Latino/Hispanic & 3.6 & 5.3 & 0.0 & 5.3 & & \\
\hline College level or above, $\%$ & 98.2 & 94.7 & 100.0 & 100.0 & $1.93(2)$ & 0.38 \\
\hline Income, $\%$ & & & & & $5.15(6)$ & 0.52 \\
\hline$<\$ 75,000$ & 14.8 & 10.5 & 23.5 & 11.1 & & \\
\hline$\$ 75,000-\$ 124,999$ & 22.2 & 26.3 & 29.4 & 11.1 & & \\
\hline$\$ 125,000-\$ 149,999$ & 13.0 & 10.5 & 5.9 & 22.2 & & \\
\hline$\$ 150,000+$ & 50.0 & 52.6 & 41.2 & 55.6 & & \\
\hline
\end{tabular}


TABLe 2: Continued.

\begin{tabular}{lcccccc}
\hline Characteristic & All & Usual care & Coach-led & Self-directed & $F / \chi^{2}($ degree of freedom) & $P$ value \\
\hline Weight, kg & $113.4 \pm 18.2$ & $116.0 \pm 20.0$ & $111.6 \pm 15.0$ & $112.6 \pm 19.4$ & $0.29(2,52)$ \\
Waist, cm & $120.1 \pm 10.9$ & $121.9 \pm 11.7$ & $117.3 \pm 9.7$ & $120.4 \pm 11.4$ & $0.78(2,52)$ \\
Fasting plasma glucose, $\mathrm{mg} / \mathrm{dL}$ & $98.7 \pm 8.6$ & $98.7 \pm 8.7$ & $98.7 \pm 9.3$ & $98.8 \pm 8.3$ & $0.00(2,52)$ \\
Prediabetes, \% & 54.5 & 52.6 & 58.8 & 52.6 & 0.46 \\
Metabolic syndrome, \% & 98.2 & 100.0 & 94.1 & 100.0 & $0.18(2)$ & 0.99 \\
Prediabetes and metabolic syndrome, \% & 52.7 & 52.6 & 52.9 & 52.6 & 0.91 \\
\hline
\end{tabular}

${ }^{\mathrm{a}}$ Plus-minus values are means $\pm \mathrm{SD}$.

incremental benefit over the self-directed intervention for participants with a baseline BMI of $30 \leq 35$, although they led greater reductions in percentage weight loss and BMI (coach-led) or waist circumference (self-directed) compared with usual care. These results imply that the self-directed intervention can be an effective and efficient alternative to the coach-led intervention for overweight individuals with prediabetes and/or metabolic syndrome but that individuals with the same cardiometabolic risk factors who are moderately or severely obese may only benefit from the latter, more structured approach. These findings add to recent evidence that suggests a structured, intensive-and yet practicallifestyle intervention is indicated for increased degree of obesity $[10,21]$, as opposed to a less structured, self-directed approach.

Previously, in the absence of empirical evidence, lifestyle intervention was thought to be ineffective in severely obese individuals [22] but has recently been recognized as a promising approach among this subpopulation [3, 8-12], especially given the risk of postoperative complications, recidivism, and limited reach of surgical options $[4,5,8]$. Modest weight loss for individuals who are overweight or obese (5\%-10\% reduction in total body weight) has been shown to produce health benefits such as improvement in blood pressure, cholesterol and dysglycemia [19, 23, 24] and was achieved among all participants in the coach-led intervention, including those whose baseline BMI was $\geq 35$. A randomized controlled trial by Goodpaster et al. showed that an intensive behavioral weight-loss intervention was effective for severely obese adults and that modest weight reduction, even despite persistent severe obesity, significantly improved cardiovascular risk factors in this population [9]. A secondary analysis of data from the Look AHEAD trial found that nearly $40 \%$ of severely obese participants in the intensive, DPP-like lifestyle intervention lost $\geq 10 \%$ of initial weight at 1 year [8], and $26 \%$ were able to maintain this weight loss at year 4 [11].

This is the first study, to our knowledge, that suggests the potential effectiveness of a coach-led, technology-enhanced DPP translation intervention in reducing obesity among adults at high risk of Type 2 diabetes and CVD with a BMI of 35 or above. This is a growing segment of the overall population for which surgery is currently recommended; however, surgery cannot be the only solution to an epidemic. It is imperative that alternative strategies are developed that are effective, accessible, and affordable with potential for broad reach and impact. Equally important, our study suggests that the low cost, self-directed intervention can be a viable alternative to the coach-led intervention for high-risk adults with a BMI less than 35 . The increased efficiency and reach of the self-directed intervention makes it an appealing public health intervention strategy.

Future studies are needed to explore factors that may modify or mediate the effectiveness of lifestyle interventions among persons with moderate or severe obesity. Potential effect modifiers include sociodemographic characteristics (e.g., sex, race/ethnicity, and education), comorbidity (e.g., severity of coexisting chronic conditions), and community/societal resources (e.g., accessibility of grocery stores or farmers market, neighborhood walkability, and social norms). Possible effect mediators include outcome expectancy, self-efficacy, social support, and self-monitoring, which are theory-based variables that have been shown to predict weight loss in diverse populations $[25,26]$.

The present findings should be interpreted with consideration of several study limitations. This was a secondary data analysis, and all findings warrant replication in future confirmatory research. The sample size for each BMI-bytreatment subgroup was small, and the trial duration was only 15 months. However, the effect size confidence intervals and data consistency across the three obesity outcome measures suggest that the E-LITE coach-led intervention may have clinically significant benefits beyond usual care for moderately and severely obese adults at high cardiometabolic risk that are worth further investigation in fully-powered, longerterm studies. Also, the generalizability of the current findings may be limited by a rather homogenous study sample in terms of race/ethnicity and socioeconomic status, and participants were recruited from a single primary care clinic. Future research is needed to confirm the generalizability of our findings to more ethnically and socioeconomically diverse populations.

\section{Conclusion}

In conclusion, baseline obesity status may influence behavioral weight loss treatment effectiveness. Less resource intensive approaches are perhaps adequate for individuals with lower baseline BMI in the overweight and obesity continuum, whereas the incremental benefit of more intensive, structured lifestyle change programs may not be evident except for those with higher BMI indicative of moderate or severe obesity. If confirmed in future definitive study, these findings would suggest that researchers and clinicians should take 
an individual's baseline BMI into account when developing or recommending a weight-loss treatment strategy. Understanding how to best allocate healthcare resources in weightloss treatment may ultimately result in improved quality and affordability of obesity care.

\section{Acknowledgments}

The content is solely the responsibility of the authors and does not necessarily represent the official views of any of the funding agencies. No sponsor or funding source had a role in the design or conduct of the study; collection, management, analysis, or interpretation of the data; or preparation, review, or approval of the paper. All authors comply with the editorial policies and have no conflict of interests. Kristen M. J. Azar and Jun Ma conceived the present study and interpreted data analysis. Lan Xiao analyzed data. All authors were involved in writing the paper and had final approval of the submitted and published versions. The E-LITE study was supported by Grant R34DK080878 from the National Institute of Diabetes and Digestive and Kidney Diseases, a Scientist Development Grant Award (0830362N) from the American Heart Association, and internal funding from the Palo Alto Medical Foundation Research Institute. The authors wish to thank the participants and their families who made the E-LITE trial possible. They would also like to acknowledge the Diabetes Prevention Support Center (DPSC) of the University of Pittsburgh for training and supporting the Group Lifestyle Balance program; the E-LITE coach-led and self-directed interventions were derived from this material.

\section{References}

[1] K. M. Flegal, D. Carroll, B. K. Kit, and C. L. Ogden, "Prevalence of obesity and trends in the distribution of body mass index among US adults, 1999-2010," Journal of the American Medical Association, vol. 307, no. 5, pp. 491-497, 2012.

[2] E. A. Finkelstein, O. A. Khavjou, H. Thompson et al., "Obesity and severe obesity forecasts through 2030," American Journal of Preventive Medicine, vol. 42, no. 6, pp. 563-570, 2012.

[3] L. E. Burke and J. Wang, "Treatment strategies for overweight and obesity," Journal of Nursing Scholarship, vol. 43, no. 4, pp. 368-375, 2011.

[4] H. Buchwald and D. M. Oien, "Metabolic/bariatric surgery worldwide 2008," Obesity Surgery, vol. 19, no. 12, pp. 1605-1611, 2009.

[5] D. B. Sarwer, R. J. Dilks, and L. West-Smith, "Dietary intake and eating behavior after bariatric surgery: threats to weight loss maintenance and strategies for success," Surgery for Obesity and Related Diseases, vol. 7, no. 5, pp. 644-651, 2011.

[6] M. Martin, A. Beekley, R. Kjorstad, and J. Sebesta, "Socioeconomic disparities in eligibility and access to bariatric surgery: a national population-based analysis," Surgery for Obesity and Related Diseases, vol. 6, no. 1, pp. 8-15, 2010.

[7] E. S. le Blanc, E. O’Connor, E. P. Whitlock, C. D. Patnode, and T. Kapka, "Effectiveness of primary care-relevant treatments for obesity in adults: a systematic evidence review for the U.S. preventive services task force," Annals of Internal Medicine, vol. 155, no. 7, pp. 434-447, 2011.
[8] J. L. Unick, D. Beavers, J. M. Jakicic et al., "Effectiveness of lifestyle interventions for individuals with severe obesity and type 2 diabetes: results from the Look AHEAD trial," Diabetes Care, vol. 34, no. 10, pp. 2152-2157, 2011.

[9] B. H. Goodpaster, J. P. DeLany, A. D. Otto et al., "Effects of diet and physical activity interventions on weight loss and cardiometabolic risk factors in severely obese adults: a randomized trial," Journal of the American Medical Association, vol. 304, no. 16, pp. 1795-1802, 2010.

[10] J. W. Anderson, L. Grant, L. Gotthelf, and L. T. P. Stifler, "Weight loss and long-term follow-up of severely obese individuals treated with an intense behavioral program," International Journal of Obesity, vol. 31, no. 3, pp. 488-493, 2007.

[11] J. L. Unick, D. Beavers, D. S. Bond et al., "The long-term effectiveness of a lifestyle intervention in severely obese individuals," American Journal of Medicine, vol. 126, no. 3, pp. 236.e2-242.e2, 2013.

[12] G. L. Blackburn, S. Wollner, and S. B. Heymsfield, "Lifestyle interventions for the treatment of class III obesity: a primary target for nutrition medicine in the obesity epidemic," American Journal of Clinical Nutrition, vol. 91, no. 1, pp. 289S-292S, 2010.

[13] M. K. Kramer, A. M. Kriska, E. M. Venditti et al., “Translating the diabetes prevention program. A comprehensive model for prevention training and program delivery," American Journal of Preventive Medicine, vol. 37, no. 6, pp. 505-511, 2009.

[14] Diabetes Prevention Support Center, "Group lifestyle balance materials," in A Modification of the Diabetes Prevention Program's Lifestyle Change Program, University of Pittsburgh, Pittsburgh, Pa, USA, 2011.

[15] J. Ma, V. Yank, L. Xiao et al., "Translating the diabetes prevention program lifestyle intervention for weight loss into primary care: a randomized trial," JAMA Internal Medicine, vol. 173, no. 2, pp. 113-121, 2013.

[16] J. Ma, A. C. King, S. R. Wilson, L. Xiao, and R. S. Stafford, "Evaluation of lifestyle interventions to treat elevated cardiometabolic risk in primary care (E-LITE): a randomized controlled trial," BMC Family Practice, vol. 10, article 71, 2009.

[17] NIH and National Heart Lung Blood Institute, Clinical Guidelines on the Identification, Evaluation, and Treatment of Overweight and Obesity in Adults: The Evidence Report, DHHS, Public Health Service, Rockville, Md, USA, 1998.

[18] D. Hunt, "Diabetes: foot ulcers and amputations," Clinical Evidence, vol. 1, p. 602, 2009.

[19] Diabetes Prevention Program Research Group, “The Diabetes Prevention Program (DPP): description of lifestyle intervention," Diabetes Care, vol. 25, no. 12, pp. 2165-2171, 2002.

[20] Diabetes Prevention Program Research Group, "10-year followup of diabetes incidence and weight loss in the Diabetes Prevention Program Outcomes Study," The Lancet, vol. 374, no. 9702, pp. 1677-1686, 2009.

[21] J. W. Anderson, S. B. Conley, and A. S. Nicholas, “One hundredpound weight losses with an intensive behavioral program: changes in risk factors in 118 patients with long-term followup," American Journal of Clinical Nutrition, vol. 86, no. 2, pp. 301-307, 2007.

[22] "Clinical guidelines on the identification, evaluation, and treatment of overweight and obesity in adults-the evidence report. National Institutes of Health," Obesity Research, vol. 6, supplement 2, pp. 51S-209S, 1998.

[23] G. Blackburn, "Effect of degree of weight loss on health benefits," Obesity Research, vol. 3, supplement 2, pp. 211s-216s, 1995. 
[24] "The Diabetes Prevention Program. Design and methods for a clinical trial in the prevention of type 2 diabetes," Diabetes Care, vol. 22, no. 4, pp. 623-634, 1999.

[25] A. Bandura, Social Foundations of Thought and Action: A Social Cognitive Theory, Prentice Hall, Englewood Cliffs, NJ, USA, 1986.

[26] A. L. Palmeira, P. J. Teixeira, T. L. Branco et al., "Predicting short-term weight loss using four leading health behavior change theories," International Journal of Behavioral Nutrition and Physical Activity, vol. 4, article 14, 2007. 


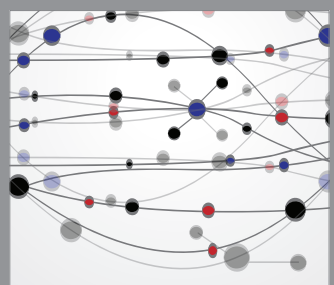

The Scientific World Journal
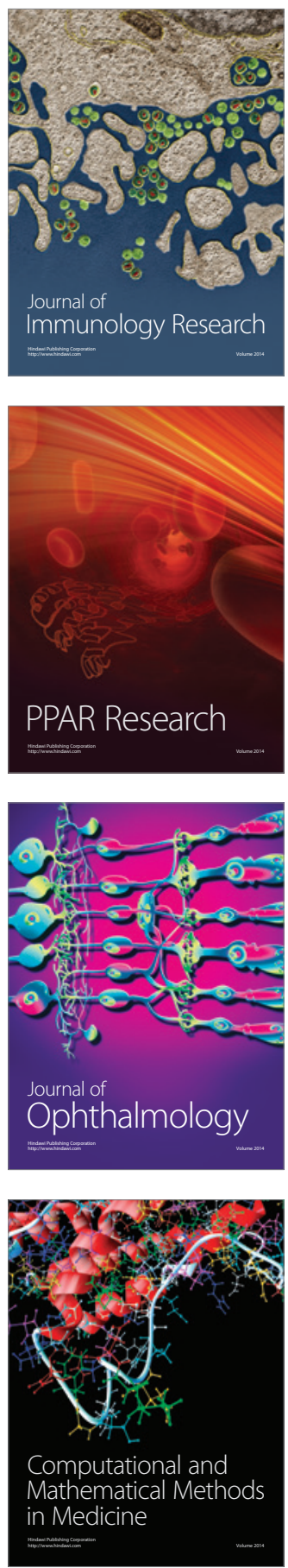

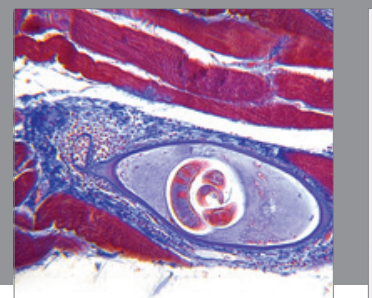

Gastroenterology

Research and Practice
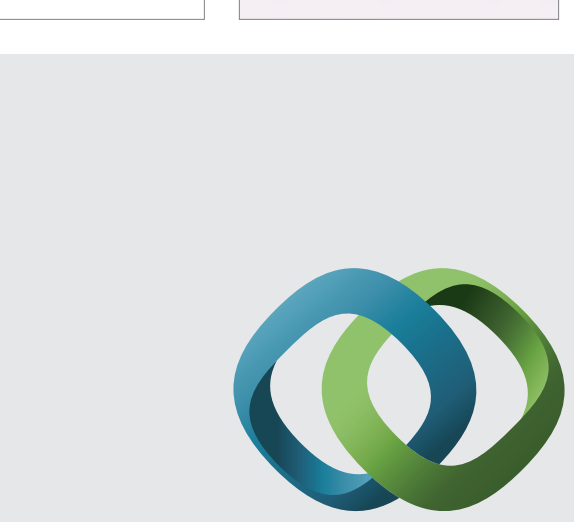

\section{Hindawi}

Submit your manuscripts at

http://www.hindawi.com
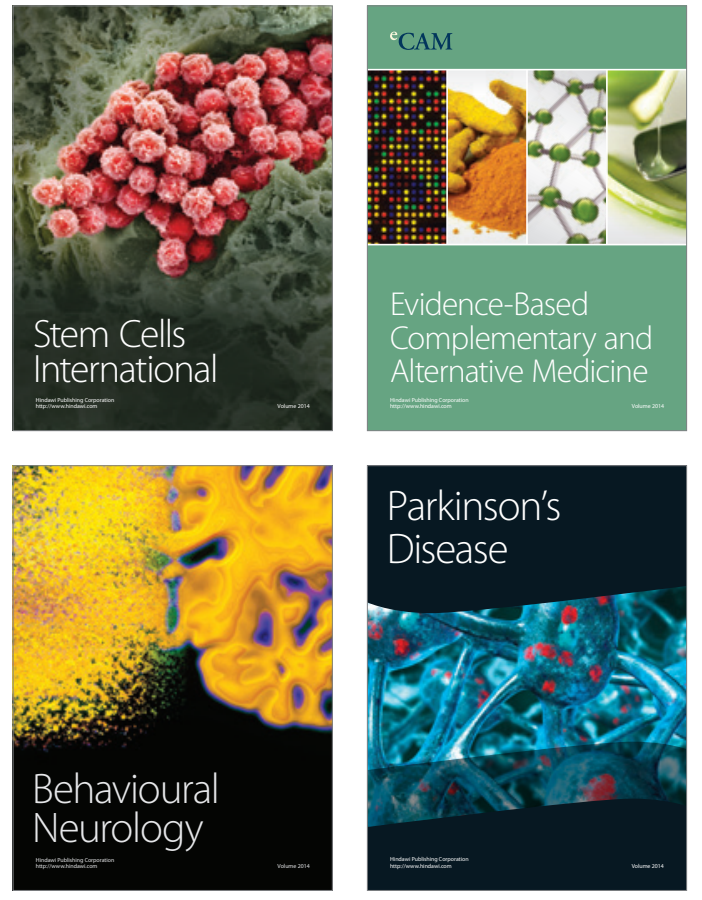
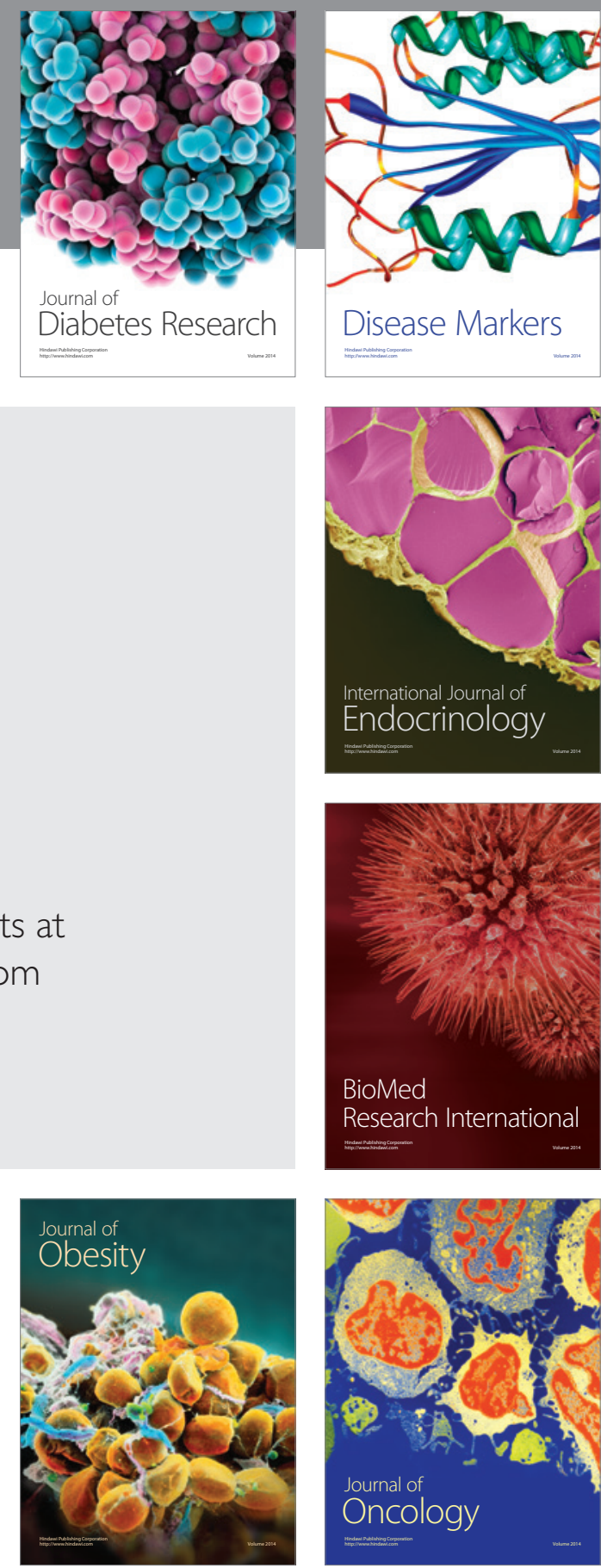

Disease Markers
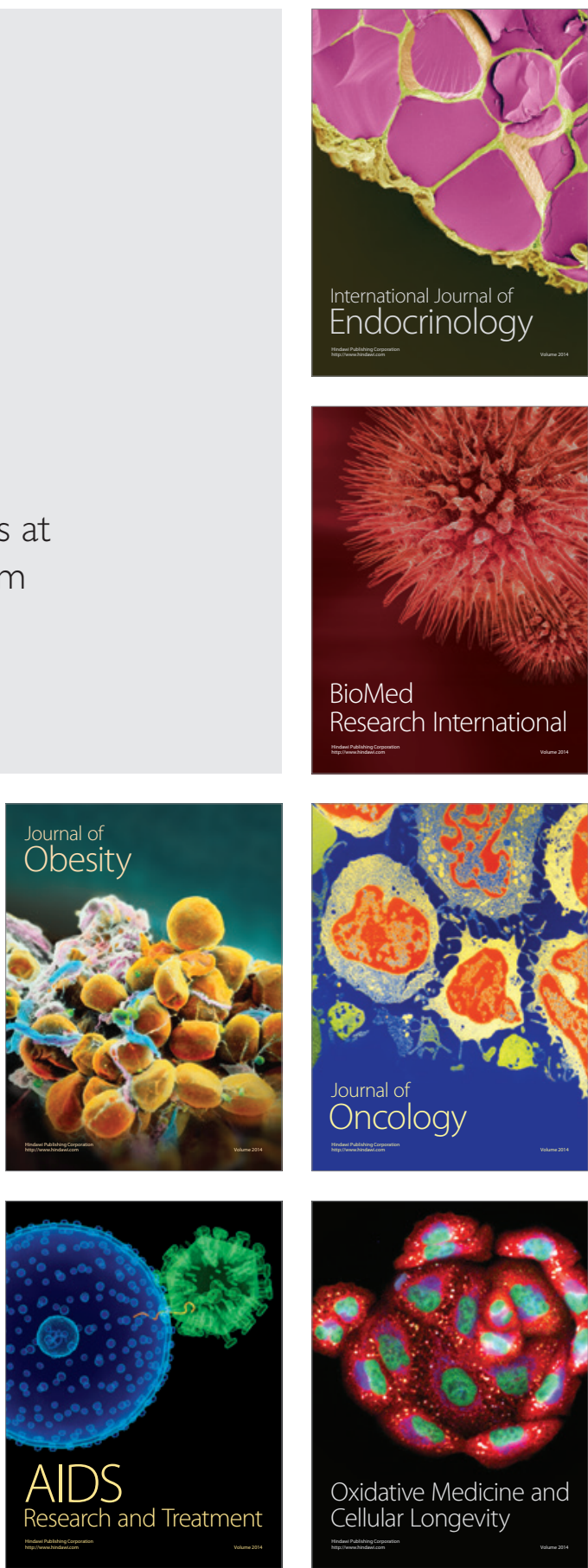